\title{
Aspectos clínicos e neuropatológicos da síndrome de Wernicke-Korsakoff
}

\section{Clinical and neurophatological aspects of Wernicke-Korsakoff syndrome}

\author{
Carlos Zubaran, Jefferson Fernandes, Fernanda Martins, Janete Souza, Rita Machado e \\ Maria Cadore \\ Departamento de Bioquímica da Universidade Federal do Rio Grande do Sul. Porto Alegre, RS - \\ Brasil (C.Z., M.C.); Hospital São Lucas da Pontifícia Universidade Católica do Rio Grande do Sul. \\ Porto Alegre, RS - Brasil (J.F.); Faculdade de Medicina da Pontifícia Universidade Católica do Rio \\ Grande do Sul. Porto Alegre, RS - Brasil (F.M., J.S., R.M.)
}

\begin{abstract}
Resumo
O abuso de álcool é um dos mais sérios problemas de saúde pública e a síndrome de Wernicke-Korsakoff é uma das mais graves conseqüências do alcoolismo. Esta patologia é infreqüentemente diagnosticada nas suas apresentações menos evidentes, razão pela qual uma abordagem diagnóstica apropriada é importante passo para seu tratamento. Entre as novas propostas farmacológicas, está a reposição dos níveis de tiamina, embora isto seja insuficiente para prevenir o declínio psicológico de um grande número de pacientes. O impacto cognitivo da patologia é derivado da interação entre neurotoxicidade alcóolica, deficiência de tiamina e suscetibilidade pessoal. São descritos, a história, a epidemiologia e os achados clínicos e neuropatológicos, bem como alguns aspectos de tratamento e prognóstico da síndrome de Wernicke-Korsakoff.
\end{abstract}

Encefalopatia de Wernicke. Transtorno amnésico alcoólico. Alcoolismo, complicações.

\begin{abstract}
Alcohol abuse is one of most serious problems in public health and the Wernicke-Korsakoff syndrome one of the gravest consequences of alcoholism. The pathology is often undiagnosed in its less evident presentations, therefore an accurate diagnostic approach is a critical step in planning treatment. Besides new pharmacological proposals, treatment is based on the restoration of thiamine, although this is insufficient to prevent the psychological decline of a great number of patients. The cognitive impact of the pathology is derived from the interaction of alcoholic neurotoxicity, thiamine deficiency and personal susceptibility. In this article the history, epidemiology, clinical and neuropathological features of the Wernicke-Korsakoff syndrome, as well as some aspects of its treatment and prognosis, are described.
\end{abstract}

Wernicke's encephalopathy. Alcohol amnestic disorder. Alcoholism, complications. 


\section{INTRODUÇÃO}

A doença de Wernicke e a psicose de Korsakoff foram identificadas no final do século passado. A primeira é caracterizada por nistagmo, marcha atáxica, paralisia do olhar conjugado e confusão mental. Esses sintomas usualmente têm início abrupto, ocorrendo mais frequientemente em combinação. A doença de Wernicke está associada com deficiência nutricional, ocorrendo especialmente em alcoolistas.

A psicose de Korsakoff é uma desordem mental na qual a memória de retenção está seriamente comprometida em um paciente até então sadio. Esta desordem está também associada ao alcoolismo e à deficiência nutricional.

O complexo de sintomas abrangendo o comprometimento do aprendizado e da memória, bem como as manifestações da doença de Wernicke é apropriadamente designado de síndrome de WernickeKorsakoff.

O objetivo do presente trabalho é descrever alguns aspectos sobre o tratamento e prognóstico da síndrome de Wernicke-Korsakoff.

\section{HISTÓRICO}

Em 1881, Carl Wernicke descreveu pela primeira vez uma patologia de início súbito, caracterizada por paralisia dos movimentos oculares, marcha atáxica e confusão mental. Observou esses sintomas em três pacientes, dois alcoolistas e uma paciente com vômitos persistentes após ingestão de ácido sulfúrico, que se apresentaram com estupor progressivo, evoluindo para a coma e morte. As alterações patológicas descritas por Wernicke foram hemorragias na substância cinzenta ao redor do terceiro e quarto ventrículos e do aqueduto de Sylvius, quando denominou a patologia de "polioenncephalitis hemorrhagica superioris".

Em 1852, Magnus Huss fez menção a um distúrbio de memória em alcoolistas, elucidado mais tarde, entre 1887 a 1891 , pelo psiquiatra russo S. S. Korsakoff, que ressaltou a relação entre a polineuropatia e a desordem de memória, como sendo "duas faces de uma mesma doença"”.

O postulado de que uma única causa é responsável pela doença de Wernicke e a psicose de Korsakoff foi feito inicialmente por Murawieff em $1897^{2}$.

\section{EPIDEMIOLOGIA}

Em estudo prospectivo de necrópsia, a prevalência da síndrome de Wernicke-Korsakof, em Sidney, Austrália, foi 2,1\% em adultos com mais de 15 anos de idade, abrangendo um amplo espectro de padrões socioeconômicos e culturais ${ }^{18}$. Outros estudos semelhantes incluem o Oeste australiano: $2,8 \%{ }^{15}$, Nova Iorque: $1,7 \%^{8}$, Oslo: $0,8 \%{ }^{51}$ e Brasil: $2,2 \%^{29}$. Os valores sobem para $15 \%$ em população psiquiátrica internada ${ }^{55}$ e para $24 \%$ em mendigos ${ }^{11}$.

Um estudo piloto de 31 mortes consecutivas, ralacionadas ao abuso de álcool, dentro de um período de 8 meses, revelou encefalopatia de Wernicke em 17 casos. A análise dos dados clínicos mostrou que o distúrbio do estado mental foi o achado mais comum e os sinais neurológicos estavam presentes em somente 2 dos 17 casos, mostrando que a encefalopatia de Wernicke pode facilmente ser subestimada como causa de deterioração no estado mental ${ }^{38}$.

\section{ASPECTOS CLÍNICOS}

A tríade clássica descrita por Wernicke é composta de oftalmoplegia, ataxia e distúrbio mentais e de consciência ${ }^{54}$. As anormalidades oculares consistem em nistagmo, horizontal ou vertical, paralisia ou paresia dos músculos retos externos e do olhar conjugado, sendo comuns achados como diplopia e estrabismo convergente. Em estágios avançados da doença, pode ocorrer miose e não reatividade pupilar. Pode ocorrer discreta hemorragia retiniana, mas papiledema é raro ${ }^{49}$.

A ataxia é de marcha e postural, sendo que nos estágios agudos da doença pode inviabilizar a deambulação ou postura sem suporte. Em graus mais brandos da patologia, os distúrbios podem ser lentidão de uma marcha incerta ou postura de base ampla.

Os distúrbios de consciência e de estado mental ocorrem principalmente como um estado confusional global, no qual o paciente está apático, desatento e com mínima expressão verbal espontânea. Os distúrbios de consciência e estado mental ocorrem em $10 \%$ dos pacientes. Com a pronta reposição de tiamina o paciente recobra rapidamente o estado de alerta e a tenacidade. Outros pacientes, em menor proporção, mostram sinais de abstinência alcoólica, com alucinações, agitação, alteração da percepção e hiperatividade autonômica. Embora estupor e coma sejam estados raros, um grupo de pacientes pode apresentar tais manifestações da doença de Wernicke, podendo evoluir para morte se não tratado.

O estado amnésico característico da psicose de Korsakoff é marcado por uma lacuna permanente na memória do paciente. O principal aspecto da desordem amnésica é o defeito do aprendizado (amnésia anterógrada) e perda da memória passada (amnésia retrógrada). A memória imediata está intacta, mas a 
memória de curto prazo está comprometida. O defeito de aprendizado é o aspecto que leva à incapacitação do paciente na sociedade, o qual fica apto para executar somente tarefas simples e habituais. A alteração deve-se à codificação defeituosa no momento do aprendizado original ao invés de um defeito exclusivo no mecanismo de recuperação ${ }^{28}$. A memória a longo prazo parece ser mantida através da rede neural multifocal, mais do que as custas de pontos anatômicos específicos, uma vez que um paciente com lesões severas tidas como específicas para síndrome de Wernicke-Korsakoff provou ter memória excelente ${ }^{4}$.

Além do comprometimento de memória, envolvendo sobretudo a evocação de material verbal e não verbal $^{41}$, os pacientes com Korsakoff apresentam desempenho inferior aos alcoolistas não-Korsakoff nos testes que avaliam funções do lobo frontal, sugerindo hipofrontalidade ${ }^{25}$.

A confabulação é um achado característico da psicose de Korsakoff . Na fase inicial da patologia, o quadro confusional é severo e a confabulação é evidente e significativa. Na fase convalescente, o paciente lembra fragmentos de experiências passadas de forma distorcida. É controversa a afirmação de que a confabulação é um recurso utilizado pelo paciente para tentar diminuir o embaraço causado pelo déficit de memória.

A neuropatia periférica está comumente associada com a doença de Wernicke-Korsakoff. Alterações cardiovasculares ocorrem habitualmente como taquicardia, hipotensão postural e anormalidades eletrocardiográficas, que resolvem após a administração de tiamina. Em estágios crônicos da doença, os pacientes podem apresentar capacidade diminuída para a discriminação entre odores.

A psicose de Korsakoff pode ocorrer nos tumores do terceiro ventrículo, infarto ou ressecção cirúrgica do lobo temporal ou como seqüela de encefalite por herpes simples ${ }^{2}$. A síndrome de WernickeKorsakoff, associada com deficiência de tiamina, tem sido descrita em pacientes sob diálise, AIDS, hyperemesis gravidarum e após gastroplastia para manejo de obesidade ${ }^{32}$.

\section{NEUROPSICOLOGIA}

Há vários modelos propostos para a compreensão dos déficits neuropsicológicos ${ }^{44}$. Pela hipótese do hemisfério direito, os processos psicológicos sob a dominância deste hemisfério, como habilidades visual e espacial, percepção e motricidade, teriam suscetibilidade aumentada aos efeitos neurotóxicos do álcool ${ }^{40,42}$. Na hipótese de disfunção do lobo fron- tal, o comprometimento da abstração e da capacidade para a resolução de tarefas seriam devidos aos efeitos do álcool no lobo frontal ${ }^{40,42}$. No entanto, a hipótese neurobiológica corrente advoga uma disfunção cerebral generalizada para explicar o padrão inespecífico e altamente variável do comprometimento cognitivo ${ }^{42,43}$.

Usando o Wiscosin Card Sorting Test (WCST) e testes de memória em pacientes com Korsakoff, pacientes amnésicos sem Korsakoff, pacientes com ressecção de lobo frontal e controles, foi verificado que o comprometimento de memória per se não poderia ser responsável pelas dificuldades na resolução de problemas dos pacientes com Korsakoff ${ }^{26}$.

\section{NEUROIMAGEM}

As avaliações da encefalopatia de Wernicke por tomografia computadorizada e ressonância magnética revelam lesões na porção medial do tálamo e mesencéfalo, dilatação do terceiro ventrículo e atrofia dos corpos mamilares ${ }^{56}$. Medições por tomografia computadorizada revelam que o terceiro ventrículo e os ventrículos laterais são mais dilatados em pacientes com psicose de Korsakoff do que em alcoolistas isentos desta patologia. $\mathrm{O}$ alargamento da fissura inter-hemisférica entre os lobos frontais é particularmente marcado ${ }^{31}$. Alterações macroscópicas nos corpos mamilares têm sido documentadas durante a vida por ressonância nuclear magnética ${ }^{7}$.

O retraimento ("shrinkage") cerebral é detectável em uma alta proporção de pacientes alcoolistas, com dilatação ventricular e alargamento de fissuras e sul$\cos$ sobre os hemisférios ${ }^{46,48}$. Foi observado que o uso corrente de álcool desempenha papel importante nesta patologia, uma vez que as tomografias computadorizadas dos membros dos Alcoólatras Anônimos (AA) mostraram maior semelhança com os exames de indivíduos-controles hígidos do que com usuários de álcool, embora algum grau de dilatação foi encontrada em certos $\operatorname{casos}^{24,30}$.

\section{NEUROQUÍMICA}

A absorção de tiamina é prejudicada pela deficiência nutricional e pelo álcool, dificultando o tratamento de alcoolistas. A situação é freqüentemente agravada pela doença hepática subjacente, que leva à redução dos estoques corporais e à diminuição do metabolismo de tiamina ${ }^{50}$. A disfunção hepática pode também acentuar os efeitos tóxicos do álcool sobre o cérebro, possivelmente através de um desequilíbrio no metabolismo dos aminoácidos ${ }^{1}$. 
A suscetibilidade pessoal à síndrome de Wernicke-Korsakoff pode estar relacionada às diferenças individuais nos sistemas enzimáticos da tiamina. Diferentes níveis de afinidade foram encontrados entre o pirofosfato de tiamina (TPP), que age como coenzima e a transcetolase, uma enzima relacionada com o metabolismo da glicose no cérebro ${ }^{14}$.

Apesar disto, poucas evidências permanecem para suportar a hipótese de uma anormalidade genética da trascetolase nos pacientes com Wernicke-Korsakoff $^{3}$, sugerindo a participação de fatores não genéticos para explicar as diferenças entre estes pacientes e os controles hígidos ${ }^{34}$. Em um estudo imunoquímico e enzimático com fibroblastos de pacientes com síndrome Wernicke-Korsakoff, a transcetolase apresentou-se cataliticamente defeituosa, mas imunoquimicamente normal ${ }^{27}$.

É notável que em torno de um terço dos pacientes parecem ser resistentes ao desenvolvimento da patologia, a despeito do consumo substancial de álcool, sugerindo uma vulnerabilidade altamente variável para a patologia ${ }^{31}$.

\section{ACHADOS FISIOLÓGICOS}

Há evidência de uma associação entre o déficit cognitivo e diminuição do fluxo hemisférico cerebral, bem como entre anormalidades regionais e perdas cognitivas específicas. O tempo de trânsito vascular cerebral dos pacientes com doença de Alzheimer e psicose de Korsakoff é lentificado ${ }^{23}$.

Fluxo sangüíneo cerebral diminuído foi encontrado em pacientes alcoolistas crônicos, com melhora em todas as estruturas corticais e subcorticais de pacientes com psicose de Korsakoff após tratamento com tiamina e abstinência alcoólica ${ }^{20}$.

\section{PATOLOGIA}

O achado mais comum nos estudos de necrópsia são alterações microscópicas nos corpos mamilares ${ }^{8,16}$.

Buscando avaliar o impacto do consumo "moderado" de álcool no cérebro, pesquisadores avaliaram medidas cerebrais como peso cerebral, espaço pericerebral e volume ventricular de bebedores moderados (30-80g de álcool/dia), alcoolistas (mais de $80 \mathrm{~g}$ de álcool/dia), alcoolistas com cirrose, alcoolistas com encefalopatia de Wernicke e grupo-controle de abstêmios ou bebedores de até $20 \mathrm{~g}$ álcool/dia. Embora não tenham encontrado diferença estatisticamente significativa entre as medidas cerebrais de bebedores "moderados" e grupo-controle, há uma tendência sugerindo perda de tecido cerebral, com peso cerebral reduzido, aumento do volume ventricular e do espaço pericerebral, sendo maior a perda de substância branca ${ }^{17}$.

A substância branca é formada de aproximadamente $70 \%$ de água, $20 \%$ de lipídios e $10 \%$ de proteína ${ }^{39}$, sendo que a maioria destes dois últimos elementos está combinada na forma de membranas (mielina particularmente). Lesões desmielinizantes mostram aumento do conteúdo de água e decréscimo no conteúdo de lipídio, invertendo o processo que ocorre durante a maturação cerebral ${ }^{12}$. $\mathrm{O}$ padrão de alteração dos conteúdos de água e de lipídio da substância branca cerebral de pacientes alcoolistas é semelhante ao padrão de alteração do envelhecimento e ao padrão de lesões desmielinizantes observados no estudo supracitado ${ }^{17}$.

Ratos expostos ao álcool por cinco meses apresentaram uma redução significativa das ramificações dendríticas no hipocampo, que reverteu após dois meses de abstinência ${ }^{37}$.

Estudos clínicos e neuroradiológicos indicam que déficits clínicos e retraimento cerebral ("brain shrinkage") são reversíveis em uma proporção de alcoolistas, preferencialmente jovens, seguindo um período prolongado de abstinência alcoólica ${ }^{6,47}$. Deve haver duas alterações patológicas na substância branca como resultado do abuso de álcool: um componente irreversível devido à morte neuronal, similar ao padrão observado na degeneração walleriana ${ }^{9}$ e um componente reversível, caracterizado por alterações sutis, de difícil identificação por exames histológicos de material humano ${ }^{17}$.

Em 131 casos de encefalopatia de Wernicke diagnosticados por autópsia, com maioria de alcoolistas, somente 26 pacientes foram diagnosticados durante a vida $^{16}$, levantando a possibilidade de que o dano cerebral tiamina-dependente possa existir em muitos alcoolistas antes de ser suspeitado ${ }^{31}$. A hipótese de um dano cerebral cumulativo poderia explicar por que a memória é tão notoriamente afetada em muitos alcoolistas e por que a psicose de Korsakoff não responde tão bem à tiamina. Dois tipos de lesão poderiam estar presentes muito antes de existir indícios clínicos para detectá-las: as lesões corticais, com possível perda neuronal, devido a neurotoxicidade provocada pelo álcool, e a patologia de regiões basais do cérebro, agravadas pela deficiência de tiamina ${ }^{31}$.

\section{DIAGNÓSTICO}

O diagnóstico clínico da encefalopatia de Wernicke, da psicose de Korsakoff ou da síndrome de Wernicke-Korsakoff ainda não está sob domínio médi- 
co, uma vez que foi estabelecido em somente $20 \%$ dos 131 casos constatados por um estudo de necrop$\operatorname{sia}^{16}$, mesmo tendo a maioria desses pacientes sido atendida em centros hospitalares durante o último período de suas vidas. O diagnóstico da síndrome de Wernicke-Korsakoff não é estabelecido em $25 \%$ dos casos, se os cérebros não são examinados microscopicamente ${ }^{18}$.

A patologia pode ser preterida possivelmente devido aos rígidos critérios tradicionais e às formas subclínicas da encefalopatia de Wernicke que, após episódios repetidos, pode propiciar o dano patológico típico do complexo Wernicke-Korsakoff ${ }^{19}$. As formas subclínicas da encefalopatia de Wernicke ocorrem como uma forma crônica da doença, com caráter progressivo, desenvolvendo-se com repetidos episódios clínicos agudos ou episódios subclínicos, causando danos estruturais cumulativos ao cérebro ${ }^{29}$, que estariam presentes muito antes de serem suspeitados.

Além disto, a encefalopatia de Wernicke pode ser facilmente subestimada como causa de deterioração do estado mental em pacientes alcoolistas, uma vez que a atenção médica é freqüentemente dirigida para outras patologias ligadas ao abuso de álcool ${ }^{38}$. A tríade clássica descrita por Wernicke ${ }^{54}$, composta de ataxia, oftalmoplegia e distúrbios mentais, demonstrou ser incomum, estando presente em $14,2 \%{ }^{38} \mathrm{e}$ $16,5 \%{ }^{19}$ dos casos diagnosticados em estudos de autópsia. Neste último ${ }^{19}$, o observação clínica mais comum foi desorientação, presente em $42 \%$ dos 97 casos, seguida por ataxia (37\%) e déficit de memória (30\%), sendo que $18,6 \%$ destes não tinham nenhum sinal clínico referido.

Os livros de medicina tradicionalmente utilizados pelos estudantes tendem a manter os critérios diagnósticos da tríade originalmente descrita por Werni$\mathrm{cke}^{54}$. Embora no livro texto de Harrison, de medicina interna $^{53}$, estes critérios não estejam ressaltados, a afirmação de que "é difícil de se fazer o diagnóstico clínico sem os sinais motores oculares" sugere estes como um critério diagnóstico fundamental.

No livro texto Cecil, de medicina interna ${ }^{10}$, mesmo com a importante ressalva de que a síndrome de Wernicke-Korsakoff "deve ser suspeitada e tratada em qualquer sujeito cronicamente malnutrido sofrendo de um estado confusional de início recente", há a afirmação de que "a tríade clínica de oftalmoplegia, ataxia e confusão global é característica" e de que "virtualmente todos pacientes têm marcha atáxica devido ao envolvimento cerebelar".

Para maior eficiência diagnóstica, é fundamental um alto índice de suspeita em "pacientes de risco", particularmente alcoolistas ${ }^{38}$. Vale ressaltar que coma pode ser sua única apresentação, razão pela qual todo paciente em coma de origem desconhecida deve receber tiamina ${ }^{29}$.

\section{TRATAMENTO}

O tratamento da síndrome de Wernicke-Korsakoff deve ser imediatamente iniciado com a administração de tiamina, uma vez que esta previne a progressão do doença e reverte as anormalidades cerebrais que não tenham provocado danos estruturais estabelecidos ${ }^{2}$.

Os pacientes devem ser hospitalizados e tiamina 50-100 mg deve ser administrada por via endovenosa diariamente por vários dias ${ }^{32,49}$, em função da comprometida absorção intestinal dos alcoolistas ${ }^{22}$. A hipomagnesemia pode dificultar a resposta ao tratamento e deve ser tratada com reposição apropriada ${ }^{13,52}$. A solução de tiamina deve ser recente, uma vez que pode ser inativada pelo calor ${ }^{45}$. A despeito do risco de diminuição da absorção intestinal com terapia oral, doses de 50 a $100 \mathrm{mg}$ de tiamina, três a quatro vezes por dia, devem ser instituídas por vários meses ${ }^{32}$. $\mathrm{O}$ paciente deve também aderir a uma dieta balanceada.

A recuperação da ataxia, após o tratamento com tiamina, pode ser incompleta, sugerindo um dano irreversível (neuropatológico) além do padrão reversível ("bioquímico") da doenças.

Quanto aos aspectos neuroquímicos da patologia, há estudos que indicam melhora clínica geral dos pacientes tratados com clonidina ${ }^{35,36}$ e fluvoxamine $^{33}$, sugerindo disfunção nos sistemas noradrenérgico e serotoninérgico respectivamente.

A síndrome de Wernicke-Korsakoff complica o tratamento do alcoolismo. Alcoolistas em declínio cognitivo respondem pobremente à psicoterapia e aos esforços educacionais. Entretanto, estudos longitudinais com psicometria mostram melhora contínua do estado mental durante períodos de abstinência, mas a evidência de dano neuronal deve ser levado em conta quando se planeja o tratamento ${ }^{21}$.

\section{PROGNÓSTICO}

A taxa de mortalidade é alta, variando de 10 a $20 \%$, principalmente devido a agravantes como infecção pulmonar, septicemia, doença hepática descompensada e a um estado irreversível de deficiência de tiamina ${ }^{2,32}$.

A pronta instituição do tratamento pode modificar o prognóstico da síndrome de WernickeKorsakoff. A oftalmoplegia inicial pode melhorar 
dentro de horas a dias, e o nistagmo, a ataxia e a confusão mental, dentro de dias a semanas ${ }^{32}$. Em torno de $60 \%$ dos pacientes apresentam nistagmo residual ou ataxia como seqüelas a longo prazo ${ }^{2,32}$.

Uma vez estabelecida, a síndrome de Korsakoff tem um prognóstico pobre, levando cerca de $80 \%$ dos pacientes a uma desordem crônica de memória. Evidências sugerem que os pacientes acometidos são aptos ao aprendizado de tarefas repetitivas simples envolvendo memória procedural. A recuperação dos

\section{REFERÊNCIAS BIBLIOGRÁFICAS}

1. ACKER, W.; APS, E. J.; MAJUMDAR, S. K.; SHAW, G. K.; THAMSON, A. D. The relationship between brain and liver damage in chronic alcoholic patients. J. Neurol. Neurosurg. Psychiatry, 45: 984-7, 1982.

2. ADAMS, R. D. \& VICTOR, M. In: Principles of neurology. New York, McGraw-Hill, 1989. p. 821-4.

3. BLANSJAAR, B. A.; ZWANG, R.; BLIJENBERG, B. G. No transketolase abnormalities in Wernicke-Korsakoff patients. J. Neurol. Sci., 106: 88-90, 1991.

4. BLANSJAAR, B. A. The localization of memory. Clin. Neurol. Neurosurg., 94: 36-8, 1992.

5. BUTTERWORTH, R. F. Pathophysiology of cerebelar dysfunction in the Wernicke - Korsakoff syndrome. Can. J. Neurol. Sci., 20: 123-6, 1993.

6. CARLEN, P. L.; WORTZMAN, G.; HOLGATE, R. C.; WILKINSON, D. A.; RANKIN, J. G. Reversible cerebral atrophy in recently abstinent chronic alcoholics measured by computed tomographic scans. Science, 200: 1076-8, 1978.

7. CHARNESS, M. E. \& DE LA PAZ, R. L. Mamillary body atrophy in Wernicke's encephalopathy: antemortem identification using magnetic resonance imageng. Ann. Neurol., 22: 595-600, 1987.

8. CRAVIOTO, H.; KOREIN, J.; SILBERMAN, J. Wernicke's encephalopathy: a clinical and pathological study of 28 autopsied cases. Arch. Neurol., 4: 510-9, 1961.

9. CUMINGS, J. N. Cerebral lipid biochemistry in the demyelinations. In: Cumings, J. N. \& Kremer, M. F. A., ed Biochemical aspects of neurological disease. Philadelphia, Davis Co., 1965. p. 229-51.

10. DAIMOND, I. Nutritional disorders of the nervous system. In: Wyngarden, B.; Smith Jr., L.H.; Bennet, J.C., ed. Cecil textbook of medicine. 19th ed. Philadelphia, WB Saunders, 1982 p. 2046-7.

11. DARNTON-HILL, I. \& TRUSWELL, A. S. Thiamin status of a sample of homeless clinic attenders in Sydney. Med.J. Aust., 152: 5-9, 1990.

12. DAVISON, A. N. \& WAJDA, M. Cerebral lipids in multiple sclerosis. J. Neurochem., 9: 427-32, 1962.

13. DYCKNER, T.; EK, B.; NYHLIN, H.; WESTER, P. O. Agravation of thiamine deficiency by magnesium depletion. Acta Med. Scand., 218: 129-31, 1985. sintomas amnésicos é lenta e incompleta e o grau máximo de recuperação poderá demorar um ano para acontecer ${ }^{2}$. Entretanto, uma recuperação significativa da função cognitiva pode ocorrer, dependendo de fatores como idade e abstinência contínua ${ }^{43}$, mas esta não pode ser predita acuradamente durante os estágios agudos da doença. Interessantemente, uma vez recuperado, o paciente com Korsakoff raramente solicita bebida alcoólica, mas poderá beber se esta lhe for oferecida ${ }^{2}$.
14. GREENWOOD, J.; JEYASINGHAM, M.; PRATT, O. E.; RYLE, T. R.; SHAW, G. K.; THOMSON, O. D. Heterogeneity of human erythrocyte transketolase: a preliminary report. Alcohol. Alcoholism, 19: 123-9, 1984.

15. HARPER, C. Wernicke's encephalopathy in Western Australia - a common preventable disease. Aust. Alcohol. Drug. Rev., 2: 71-3, 1982.

16. HARPER, C. G. The incidence of Wernicke's encephalopathy in Australia: a neuropathological study of 131 cases. $J$. Neurol. Neurosurg. Psychiatr., 46: 593-8, 1983.

17. HARPER, C.; KRIL, J.; DALY, J. Does a "moderate" alcohol intake damage the brain? J. Neurol. Neurosurg. Psychiatr., 51: 909-13, 1988.

18. HARPER, C.; GOLD, J.; RODRIGUEZ, M.; PERDICES, M. The prevalence of the Wernicke-Korsakoff in Sydney, Australia: a prospective necropsy study. J. Neurol. Neurosurg. Psychiatry, 52: 282-5, 1989.

19. HARPER, C. G.; GILES, M.; FINLAY-JONES, R. Clinical signs in the Wernicke-Korsakoff complex: a retrospective analysis of 131 cases diagnosed at necropsy. J. Neurol. Neurosurg. Psychiatr., 49: 341-5, 1989.

20. HATA, T.; MEYER, J. S.; TANAHASHI, N., et al. Threedimensional mapping of local cerebral perfusion in alcoholic encephalopathy with and without WernickeKorsakoff syndrome. J. Cereb. Blood Flow Metab., 7: 35-44, 1987.

21. HORVATH, T. B.; SIEVER, L. J.; MOHS, R. C.; DAVIS, K. Organic mental syndromes and disorders. In: Kaplan H. I.; Sadock, B. J., ed. Comprehensive textbook of psychiatry. Baltimore, Williams and Wilkins, p. 599-641, 1989.

22. HOYUMPA, A. M. Mechanism of vitamin deficiencies in alcoholism. Alcohol. Clin. Exp. Res., 10: 572-81, 1986.

23. HUNTER, R.; MERRICK, M. V.; FERRINGTON, C., et al. Cerebral vascular transit time in Alzheimer's disease and Korsakoff's psychosis and its relation to cognitive function. Br. J. Psychiatr., 154: 709-96, 1989.

24. JACOBSON, R. The contribution of sex and drinking history to the CT brain scan changes in alcoholics. Psychol. Med., 16: 547-59, 1986.

25. JACOBSON, R. Alcoholism, Korsakoff's syndrome and the frontal lobes. Behav. Neurol., 2: 25-38, 1989. 
26. JOYCE, E. M. \& ROBBINS, T. W. Frontal lobe function in Korsakoff and non-korsakoff alcoholics: planning and spatial working memory Neuropsychologia, 29: 709, 1991.

27. JUNG, E. H.; SHEU, K. F.; BLASS, J. P. An enzymatic and immunochemical analysis of transketolase in fibroblast from Wernicke-Korsakoff syndrome. J. Neurol. Sci., 114: 123-7, 1993.

28. KUPFERMANN, I. Learning and memory. In: Kandel, E. R., ed. Principles of neural science. 3rd ed. Connecticut, Appleton and Lange, 1991. p. 997- 1008.

29. LANA-PEIXOTO, M. A.; SANTOS, E. C.; PITTELLA, J. E. Coma and death in unrecognized Wernicke's encephalopathy - an autopsy study. Arq. Neuro-Psiquiatr, 50: 329-33, 1992.

30. LISHMAN, W. A.; JACOBSON, R. R.; ACKER, C. Brain damage in alcoholism: current concepts. Acta Med. Scand., 717: 5-17, 1987.

31. LISHMAN, W. A. Alcohol and the brain. Br. J. Psychiatr., 156: 635-44, 1990.

32. MANCALL, E. L. Nutritional disorders of the nervous system. In: Aminoff, M. J., ed. Neurology and general medicine. New York, Churchill Livingstone, 1995. p. 285 301.

33. MARTIN, P. R.; ADINOFF, B.; ECKARDT, M., J. et al. Effective pharmacotherapy of alcoholic amnesic disorder with fluvoxamine: preliminary findings. Arch. Gen. Psychiatr., 46: 617-21, 1989.

34. MCCOOL, B. A.; PLONK, S. G.; MARTIN, P. R.; SINGLETON, C. K. Cloning of human transketolase cDNAs and comparison of the nucleotide sequence of the coding region in Wernicke-Korsakoff and nom-Wernicke-Korsakoff individuals. J. Biol. Chem. , 268: 1397- 404, 1993.

35. MCENTEE, W. J. \& MAIR, R. G. Memory enhancement in korsakoff's psychosis by clonidine: further evidence for a noradrenergic deficit. Ann. Neurol., 7: 466-70, 1980.

36. MCENTEE, W. J.; MAIR, R. G.; LANGLAIS, P. J. Neurochemical pathology in Korsakoff's psychosis: implications for other cognitive disorders. Neurol., 34: 64852,1984 .

37. MCMULLEN, P. A.; SANT-CYR, J. A.; CARLEN, P. L. Morphological alterations in rat CA1 hippocampal pyramid cell dendrites resulting from chronic ethanol consumption and withdrawal. J. Comp. Neurol., 225: 111-8, 1984.

38. NAIDOO, D. P.; BRAMDEV, A.; COOPER, K. Wernicke's encephalopathy and alcohol- related disease. Postgrad. Med. J., 67: 978-81, 1991.

39. O'BRIEN, J. S. \& SAMPSON, E. L. Lipid composition of the normal human brain: grey matter,white matter and myelin. J. Lipid. Res., 6: 537-44, 1965.

40. OSCAR-BERMAN, M. Neuropsychological consequences of alcohol abuse: Questions, hypotheses, and models. In: Parsons, O. A.; Butters, N.; Nathan, P. E.; ed. Neuropsychology of alcoholism:implications for diagnosis and treatment. New York, Guilford Press, 1987. p. 256-72.

41. PARKIN, A. J.; DUNN, J. C.; LEE, C.; O'HARA, P. F., NUSSBAUM, L. Neuropsycological sequelae of Wernicke encephalopathy in a 20-year-old woman: selective impairment of a frontal memory system. Brain Cogn., 21: 1-19, 1993.

42. PARSONS, O. A. \& LEBER, W. R. The relationship between cognitive dysfunction and brain damage in alcoholics: Causal, interactive, or epiphenomenal? Alcohol. Clin. Exp. Res., 5: 326-43, 1981.

43. PARSONS, O. A. Neuropsychological consequences of alcohol abuse: Many questions - some answers. In: Parsons, O. A. Butters, N.; Nathan, P. E., ed. Neuropsychology of alcoholism: implications for diagnosis and treatment. New York, Guilford Press, 1987. p. 153-75.

44. PARSONS, O. A. \& NIXON, S. J. Neurobehavioral sequelae of alcoholism. Neurol. Clin., 11: 205-18, 1993.

45. REULER, J. B.; GIRARD, D. E.; COONEY, T. G. Current concepts: Wernicke's encephalopathy. N. Eng. J. Med., 312: 1035-9, 1985

46. RON, M. A.; ACKER, W.; SHAW, G. K.; LISHMAN, W, A. Computerized tomography of the brain in chronic alcoholism. A survey and follow-up study. Brain, 105: 497-514, 1982.

47. RON, M. A. The alcoholic brain: CT scan and psychological findings. Psychol. Med., 3: 1-33, 1983.

48. RON, M. A.; ACKER, W.; LISHMAN, W.A. Morphological abnormalities in the brains of chronic alcoholics. A clinical, psychological and computerized axial tomographic study. Acta Psychiatr. Scand., 62: 41-6.

49. SO, Y. T. \& SIMON, R. P. Deficiency diseases of the nervous system. In: Bradley, G. W.; Daroff, R.B.; Fenichel, G. M.; Marsden, C. D, ed. Neurology in clinical practice. Oxford, Butterworth-Heinemann, 1989. p. 1176-8.

50. THOMSON, A. D.; JEYASINGHAM, M. D.; PRATT, O. E.; SHAW, G. K. Nutrition and alcoholic encephalopathies. Acta Med. Scand., 717: 55-65, 1987.

51. TORVIK, A.; LINDBOE, C. F.; RODGE, S. Brain lesions in alcoholics: a neurophatological study with clinical correlations. J. Neurol. Sci., 56: 233-48, 1982.

52. TRAVIESA, D. C. Magnesiun deficiency! A possible cause of thiamne refractoriness in Wernicke-Korsakoff encephalopathy. J. Neurol. Neurosurg. Psychiatr., 37: 959-62, 1974.

53. VICTOR, M. \& MARTIN, J. Nutricional and metabolic diseases of the nervous system. In : Isselbacher, K. J.; Braunwald, E.; Wilson, J. D.; Martin, J. B.; Fauci, A. S.; Kasper, D. L. ed. Harrison's principles of internal medicine. 30 th ed. McGraw-Hill, New York, p. 2329, 1994.

54. WERNICKE, C., ed. Die äkute hamorrhagische Polioencephalitis superior. In: Lehrbuch der Gehirnkrankheiten fär Ärzte und Studierende. Kassel, Fischer, 1981, Band 2, p. 229-42.

55. YELLOWLEES, P. M. Thiamin deficiency and prevention of the Wernicke-Korsakoff syndrome. A major public health problem. Med. J. Aust., 745: 216-9, 1986.

56. YOKOTE, K.; MIYAGI, K.; KUZUHARA, S.; YAMANOUCHI, H.; YAMADA, H. Wernicke encephalopathy: follow-up study by CT and MR. $J$. Comput Assist. Tomogr., 15: 835-8, 1991. 